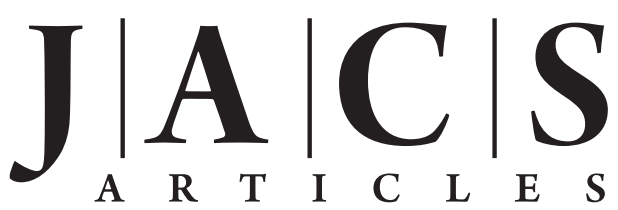

Published on Web 12/16/2009

\title{
Tuning the Extent of Chiral Amplification by Temperature in a Dynamic Supramolecular Polymer
}

\author{
Maarten M. J. Smulders, ${ }^{\dagger}$ Ivo A. W. Filot, ${ }^{\dagger}$ Janus M. A. Leenders, ${ }^{\dagger}$ Paul van der \\ Schoot, ${ }^{\ddagger}$ Anja R. A. Palmans, ${ }^{*, \dagger}$ Albertus P. H. J. Schenning, ${ }^{,, \dagger}$ and E. W. Meijer ${ }^{*, \dagger}$ \\ Laboratory of Macromolecular and Organic Chemistry, Institute for Complex Molecular \\ Systems, and Group Theory of Polymers and Soft Matter, Eindhoven University of Technology, \\ P.O. Box 513, 5600 MB Eindhoven, The Netherlands
}

Received September 30, 2009; E-mail: A.Palmans@tue.nl; A.P.H.J.Schenning@tue.nl; E.W.Meijer@tue.nl

\begin{abstract}
Here, we report on the strong amplification of chirality observed in supramolecular polymers consisting of benzene-1,3,5-tricarboxamide monomers and study the chiral amplification phenomena as a function of temperature. To quantify the two chiral amplification phenomena, i.e., the sergeants-and-soldiers principle and the majority-rules principle, we adapted the previously reported sergeants-and-soldiers model, which allowed us to describe both amplification phenomena in terms of two energy penalties: the helix reversal penalty and the mismatch penalty. The former was ascribed to the formation of intermolecular hydrogen bonds and was the larger of the two. The latter was related to steric interactions in the alkyl side chains due to the stereogenic center. With increasing temperature, the helix reversal penalty was little affected and remained rather constant, showing that the intermolecular hydrogen bonds remain intact and are directing the helicity in the stack. The mismatch penalty, however, was found to decrease when the temperature was increased, which resulted in opposite effects on the degree of chiral amplification when comparing the sergeants-and-soldiers and the majority-rules phenomena. While for the former a reduction in mismatch penalty resulted in a decrease in degree of chiral amplification, for the latter it resulted in a stronger chiral amplification effect. By combining the sergeants-and-soldiers and majority-rules phenomena in a diluted majority-rules experiment, we could further determine the effect of temperature on the degree of chiral amplification. Extending the experiments to different concentrations revealed that the relative temperature, i.e., the temperature relative to the critical temperature of elongation, controls the degree of chiral amplification. On the basis of these results, it was possible to generate a general "master curve" independent of concentration to describe the temperature-dependent majority-rules principle. As a result, unprecedented expressions of amplification of chirality are recorded.
\end{abstract}

\section{Introduction}

The helical structure is a ubiquitous motif found in natural polymers, as well as in synthetic polymers, ${ }^{1-5}$ such as polymethacrylates, polyisocyanides, polyisocyanates, and polyacetylenes. For these helical, synthetic polymers, a distinction is made between conformationally static and dynamic polymers, that is, polymers whose helicity is fixed throughout the whole polymer chain without appreciable interconversion (i.e., atropisomerization) and polymers in which both helicities occur in a single polymer chain with a fast atropisomerization. In both cases, a stereogenic center present in the monomer may favor one of the helicities, resulting in optical activity. Polyisocyanates are among the polymers that can be classified in the group of dynamic polymers, and it was exactly their dynamic character

\footnotetext{
${ }^{\dagger}$ Laboratory of Macromolecular and Organic Chemistry and Institute for Complex Molecular Systems.

Group Theory of Polymers and Soft Matter.

(1) Nakano, T.; Okamoto, Y. Chem. Rev. 2001, 101, 4013-4038.

(2) Yashima, E.; Maeda, K.; Furusho, Y.Acc. Chem. Res. 2008, 41, 11661180.

(3) Green, M. M.; Cheon, K.-S.; Yang, S.-Y.; Park, J.-W.; Swansburg, S.; Liu, W. Acc. Chem. Res. 2001, 34, 672-680.

(4) Maeda, K.; Yashima, E. Top. Curr. Chem. 2006, 265, 47-88.

(5) Yashima, E.; Maeda, K. Macromolecules 2008, 41, 3-12.
}

that allowed Green and co-workers to study chiral amplification phenomena in copolymers of isocyanate monomers. ${ }^{6-8} \mathrm{Co}-$ polymerization of chiral and achiral isocyanates led to the observation that only small amounts of the chiral monomer were required to obtain a homochiral polymer. ${ }^{9,10}$ Mixing enantiomeric monomers in different ratios afforded polyisocyanates whose helicity, as expressed by their optical activity, showed a nonlinear dependence on the enantiomeric excess (ee). ${ }^{7,11}$ The two effects that influenced the amplification of chirality were referred to as the sergeants-and-soldiers principle and the majority-rules principle. The sergeants-and-soldiers principle

(6) Green, M. M.; Park, J.-W.; Sato, T.; Teramoto, A.; Lifson, S.; Selinger, R. L. B.; Selinger, J. V. Angew. Chem.. Int. Ed. 1999, 38, 31383154.

(7) Green, M. M.; Peterson, N. C.; Sato, T.; Teramoto, A.; Cook, R.; Lifson, S. Science 1995, 268, 1860-1866.

(8) Green, M. M.; Cheon, K. S.; Yang, S. Y.; Park, J. W.; Swansburg, S.; Liu, W. Acc. Chem. Res. 2001, 34, 672-680.

(9) Gu, H.; Nakamura, Y.; Sato, T.; Teramoto, A.; Green, M. M.; Jha, S. K.; Andreola, C.; Reidy, M. P. Macromolecules 1998, 31, 63626368.

(10) Green, M. M.; Reidy, M. P. J. Am. Chem. Soc. 1989, 111, 64526454.

(11) Green, M. M.; Garetz, B. A.; Munoz, B.; Chang, H.; Hoke, S.; Cooks, R. G. J. Am. Chem. Soc. 1995, 117, 4181-4182. 


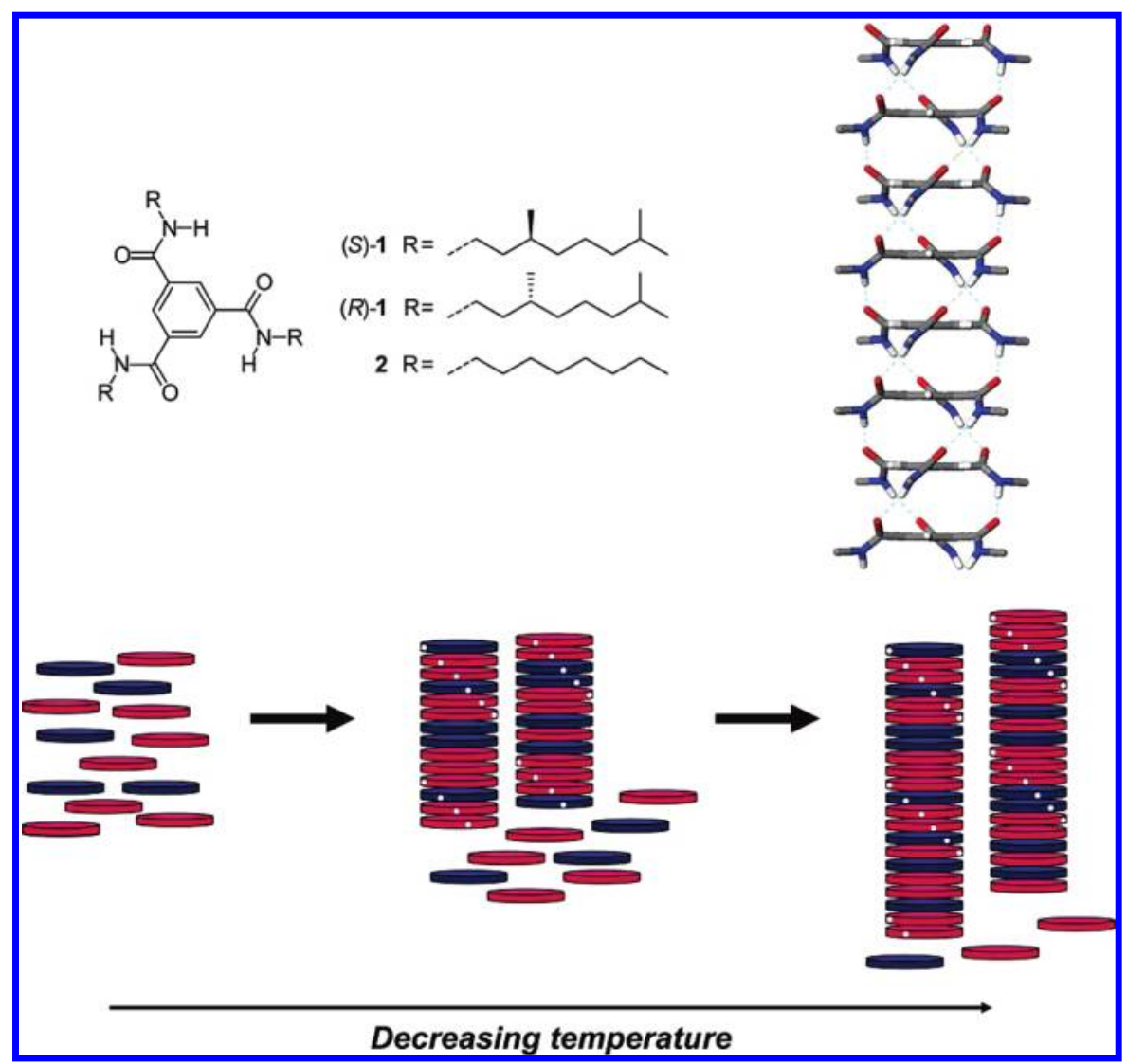

Figure 1. Structure of $C_{3}$-symmetrical chiral discotics $(S)-\mathbf{1}$ and $(R)-\mathbf{1}$ and achiral analogue $\mathbf{2}$ (top left) and the right-handed helical structure proposed for the stacking of the $C_{3}$-symmetrical discotics, based on the X-ray structure reported by Lightfoot ${ }^{32}$ (top right). For clarity the side chains were replaced by methyl groups (omitting the $\mathrm{H}$-atoms). Schematic representation of the majority-rules effect as a function of temperature (bottom). At high temperatures, only monomers are present and no chiral amplification can occur. Upon lowering the temperatures, some of the monomers are converted into stacks in which the helicity is governed by the enantiomer in excess. Further lowering of the temperature will convert practically all the monomers into helical stacks in which the majority-rules effect will remain operative.

implies control of the helicity of large numbers of cooperative achiral units, the "soldiers", by a few chiral units, the "sergeants". In the majority-rules principle, a slight excess of one enantiomer leads to a strong bias toward the helicity corresponding to the major enantiomer.

In contrast to amplification of chirality observed in a number of synthetic systems ${ }^{12-15}$ or the chiral symmetry breaking during crystallization, ${ }^{16}$ in helical polymers the net ee of the monomer does not increase, while the macromolecular chirality does. The term "amplification of chirality" in supramolecular chemistry and in the field of helical polymers implies that at the supramolecular level a full expression of a chiral superstructure exists. In contrast, in organic chemistry the term "amplification of chirality" is used when the ee of the reaction product increases relative to the substrate or catalyst used.

Interestingly, amplification of chirality has also been observed in noncovalent systems, as recently reviewed by our group. ${ }^{17}$

(12) Soai, K.; Shibata, T.; Morioka, H.; Choji, K. Nature 1995, 378, 767768.

(13) Sato, I.; Urabe, H.; Ishiguro, S.; Shibata, T.; Soai, K. Angew. Chem. Int. Ed. 2003, 42, 315-317.

(14) Mauksch, M.; Tsogoeva, S. B.; Martynova, I. M.; Wei, S. Angew. Chem., Int. Ed. 2007, 46, 393-396.

(15) Amedjkouh, M.; Brandberg, M. Chem. Commun. 2008, 3043-3045.

(16) Noorduin, W. L.; Izumi, T.; Millemaggi, A.; Leeman, M.; Meekes, H.; van Enckevort, W. J. P.; Kellogg, R. M.; Kaptein, B.; Vlieg, E.; Blackmond, D. G. J. Am. Chem. Soc. 2008, 130, 1158-1159.

(17) Palmans, A. R. A.; Meijer, E. W. Angew. Chem., Int. Ed. 2007, 46, $8948-8968$.
Prerequisites are that a chiral superstructure must exist and that the noncovalent interactions between the monomers are strong. To quantify the chiral amplification behavior for either covalent or supramolecular polymers, various models have been developed by Green et al., ,9,18-20 Selinger and Selinger, ${ }^{21-23}$ Tanaka, ${ }^{24}$ Teramoto, ${ }^{25}$ and van Gestel et al. ${ }^{26-29}$

Herein, we present our results on chiral amplification phenomena, i.e., the sergeants-and-soldiers and the majorityrules principles, in a dynamic, supramolecular polymer. The supramolecular polymer we studied is based on $C_{3}$-symmetrical trialkylbenzene-1,3,5-tricarboxamide monomers, i.e., chiral discotics $(S)-\mathbf{1}$ and $(R)-\mathbf{1}$ and achiral derivative $\mathbf{2}$ (Figure 1), one

(18) Lifson, S.; Green, M. M.; Andreola, C.; Peterson, N. C. J. Am. Chem. Soc. 1989, 111, 8850-8858.

(19) Tang, K.; Green, M. M.; Cheon, K. S.; Selinger, J. V.; Garetz, B. A. J. Am. Chem. Soc. 2003, 125, 7313-7323.

(20) Cheon, K. S.; Selinger, J. V.; Green, M. M. J.Phvs. Org. Chem. 2004, 17, 719-723.

(21) Selinger, J. V.; Selinger, R. L. B. Phvs. Rev. E 1997, 55, 1728.

(22) Selinger, J. V.; Selinger, R. L. B. Phys. Rev. Lett. 1996, 76, 58.

(23) Selinger, J. V.; Selinger, R. L. B. Macromolecules 1998, 31, 24882492.

(24) Tanaka, F. Macromolecules 2004, 37, 605-613.

(25) Teramoto, A. Prog. Polym. Sci. 2001, 26, 667-720.

(26) van Gestel, J. J. Phvs. Chem. B 2006, 110, 4365-4370.

(27) van Gestel, J.; van der Schoot, P.; Michels, M. A. J. J. Chem. Phys. 2004, 120, 8253-8261.

(28) van Gestel, J. Macromolecules 2004, 37, 3894-3898.

(29) van Gestel, J.; van der Schoot, P.; Michels, M. A. J. Macromolecules 2003, 36, 6668-6673. 
of the simplest and most studied building blocks in self-assembly. ${ }^{30-47}$ Recently, we reported on the cooperative self-assembly of $(R)-\mathbf{1}$ and 2, based on temperature-dependent $\mathrm{UV}-\mathrm{vis}$ and circular dichroism (CD) spectroscopy measurements. ${ }^{48}$ A strong, nonsigmoidal transition of the CD effect and UV absorption, probed at a wavelength characteristic for aggregation, was found upon cooling a solution of $(R)-\mathbf{1}$ in heptane. Furthermore, chiral amplification for $C_{3}$-symmetrical discotic molecules was already reported, in dilute solution by our own group ${ }^{30,35,36,48}$ as well as in an organogel system by the group of Hanabusa. ${ }^{46,49}$

We were especially interested in the role of temperature on chiral amplification, since both the degree of aggregation and the average stack length are affected by temperature. Also, the chiral amplification phenomena themselves are expected to display a nontrivial temperature dependence (Figure 1).

In covalent polymers, the effect of temperature was studied for polyisocyanates by Selinger et al. ${ }^{19,22}$ However, the effect of temperature on chiral amplification for supramolecular polymers has so far received little attention. Our group has reported on the majority-rules principle for $C_{3}$-symmetrical monomers, equipped with acylated 2,2'-bipyridine-3,3'-diamine moieties, at 20 and $50{ }^{\circ} \mathrm{C}$ in octane. ${ }^{38}$ These monomers selfassemble via an isodesmic mechanism into helical stacks. At $50^{\circ} \mathrm{C}$ a weaker majority-rules effect was observed, as compared to results at $20{ }^{\circ} \mathrm{C}$, which was attributed to the partial disassembly of the supramolecular polymer at higher temperature. However, there are no examples of the effect of temperature on chiral amplification in a supramolecular polymer that self-assembles via a cooperative mechanism. This prompted

(30) Brunsveld, L.; Schenning, A. P. H. J.; Broeren, M. A. C.; Janssen, H. M.; Vekemans, J. A. J. M.; Meijer, E. W. Chem. Lett. 2000, 292293.

(31) Matsunaga, Y.; Miyajima, N.; Nakayasu, Y.; Sakai, S.; Yonenaga, M. Bull. Chem. Soc. Jpn. 1988, 61, 207-210.

(32) Lightfoot, M. P.; Mair, F. S.; Pritchard, R. G.; Warren, J. E. Chem. Commun. 1999, 1945-1946.

(33) van Gorp, J. J.; Vekemans, J. A. J. M.; Meijer, E. W. J. Am. Chem. Soc. 2002, 124, 14759-14769.

(34) Yasuda, Y.; Iishi, E.; Inada, H.; Shirota, Y. Chem. Lett. 1996, 575576.

(35) Wilson, A. J.; Masuda, M.; Sijbesma, R. P.; Meijer, E. W. Angew. Chem. Int. Ed. 2005, 44, 2275-2279.

(36) Wilson, A. J.; van Gestel, J.; Sijbesma, R. P.; Meijer, E. W. Chem. Commun. 2006, 4404-4406.

(37) Palmans, A. R. A.; Vekemans, J. A. J. M.; Havinga, E. E.; Meijer, E. W. Angew. Chem., Int. Ed. Engl. 1997, 36, 2648-2651.

(38) van Gestel, J.; Palmans, A. R. A.; Titulaer, B.; Vekemans, J. A. J. M.; Meijer, E. W. J. Am. Chem. Soc. 2005, 127, 5490-5494.

(39) Nguyen, T.-Q.; Martel, R.; Avouris, P.; Bushey, M. L.; Brus, L.; Nuckolls, C. J. Am. Chem. Soc. 2004, 126, 5234-5242.

(40) Bushey, M. L.; Nguyen, T.-Q.; Zhang, W.; Horoszewski, D.; Nuckolls, C. Angew. Chem. Int. Ed. 2004, 43, 5446-5453.

(41) Bushey, M. L.; Hwang, A.; Stephens, P. W.; Nuckolls, C. Angew. Chem. Int. Ed. 2002, 41, 2828-2831.

(42) Matsunaga, Y.; Nakayasu, Y.; Sakai, S.; Yonenaga, M. Mol. Crust. Liq. Cryst. 1986, 141, 327-333.

(43) Hanabusa, K.; Koto, C.; Kimura, M.; Shirai, H.; Kakehi, A. Chem. Lett. 1997, 429-430.

(44) Shikata, T.; Ogata, D.; Hanabusa, K. J. Phys. Chem. B 2004, 108, $508-514$.

(45) Sakamoto, A.; Ogata, D.; Shikata, T.; Urakawa, O.; Hanabusa, K. Polvmer 2006, 47, 956-960.

(46) Ogata, D.; Shikata, T.; Hanabusa, K. J. Phys. Chem. B 2004, 108, $15503-15510$.

(47) Blomenhofer, M.; Ganzleben, S.; Hanft, D.; Schmidt, H.-W.; Kristiansen, M.; Smith, P.; Stoll, K.; Mader, D.; Hoffmann, K. Macromolecules 2005, 38, 3688-3695.

(48) Smulders, M. M. J.; Schenning, A. P. H. J.; Meijer, E. W. J. Am. Chem. Soc. 2008, 130, 606-611.

(49) Shikata, T.; Kuruma, Y.; Sakamoto, A.; Hanabusa, K. J. Phvs. Chem. B 2008, 112, 16393-16402. us to systematically study the temperature dependence of chiral amplification phenomena for the cooperative benzene-1,3,5tricarboxamide (BTA) monomers. To quantify the chiral amplification behavior as a function of temperature in a supramolecular polymer, we adapted the previously reported sergeants-and-soldiers model, developed by van Gestel, ${ }^{29}$ to include also a so-called mismatch penalty (vide infra), similarly as for the majority-rules principle. ${ }^{28}$ The insights resulted in an unprecedented temperature-dependent amplification of chirality due to the dynamic character of the cooperative supramolecular polymers.

\section{Results}

Chiral Amplification at Room Temperature. While the sergeants-and-soldiers principle was already reported for $(S)$-1 and $2,{ }^{30}$ no majority-rules experiments have so far been performed for the enantiomers $(S) \mathbf{- 1}$ and $(R)-\mathbf{1}$ in dilute solution. Therefore, initial experiments were performed to investigate this phenomenon, by adding small volumes of a solution of the $(S)$ enantiomer to a constant volume of a solution of the $(R)$ enantiomer at the same concentration in the same solvent, methylcyclohexane $(\mathrm{MCH})$, after which the $\mathrm{CD}$ spectrum was recorded. Due to the dynamic nature of the supramolecular polymer at room temperature, there is a fast exchange between stacks and monomers, as reported in our earlier study of the sergeants-and-soldiers principle. ${ }^{48}$ As a result, simple mixing of the two solutions of the different enantiomers leads to a fast setting of a new equilibrium within $1 \mathrm{~min}$, allowing the experiments to be performed in this manner.

Figure 2A shows the change in the $\mathrm{CD}$ spectrum upon consecutive additions of volumes of about $50-100 \mu \mathrm{L}$ of a 2.0 $\times 10^{-5} \mathrm{M}$ solution of $(S)-\mathbf{1}$ in $\mathrm{MCH}$ to $2 \mathrm{~mL}$ of a $2.0 \times 10^{-5}$ M solution of $(R)-\mathbf{1}$ in $\mathrm{MCH}$. Since we are mixing solutions of enantiomers at equal concentration, no change in UV-vis absorption could be discerned upon addition of the $(S)$ enantiomer. Furthermore, the shape of the CD effect does not depend on the ratio of the two enantiomers. However, the intensity of the CD effect does depend on the ee. Thus, the initially positive $\mathrm{CD}$ effect, with its maximum intensity at 223 $\mathrm{nm}$, remains constant until an ee of $40 \%$ is reached; when the ee is reduced further, the CD effect rapidly weakens in intensity, becoming zero at an ee of $0 \%$. Adding more $(S)-\mathbf{1}$ results in the appearance of a negative $\mathrm{CD}$ effect. Plotting the maximum in $C D$ effect at $223 \mathrm{~nm}$ against the ee revealed a strong nonlinear dependence, as can be seen in Figure 2B. Up to an ee of about $40 \%$ a saturated CD signal can be observed, indicative of a fully homochiral supramolecular system. In addition, commensurate with expectation, the majority-rules data in Figure 2B are pointsymmetric with respect to the origin.

To quantify these majority-rules data we used the model developed by van Gestel, which describes the majority-rules phenomenon by introduction of two free energy penalties, i.e., the helix reversal penalty (HRP) and the mismatch penalty (MMP). The former penalizes a helix reversal in a stack, whereas the latter penalizes a mismatch when a chiral monomer is introduced into a stack of its unpreferred helicity. ${ }^{28}$ This model was applied successfully previously to analyze the majorityrules phenomenon in supramolecular polymers. ${ }^{36,38}$

After converting the measured $\mathrm{CD}$ values into the dimensionless net helicity, $\eta$, we obtained a least-squares fit of the normalized majority-rules data shown in Figure $2 \mathrm{~B} .{ }^{50}$ This

(50) See Supporting Information. 


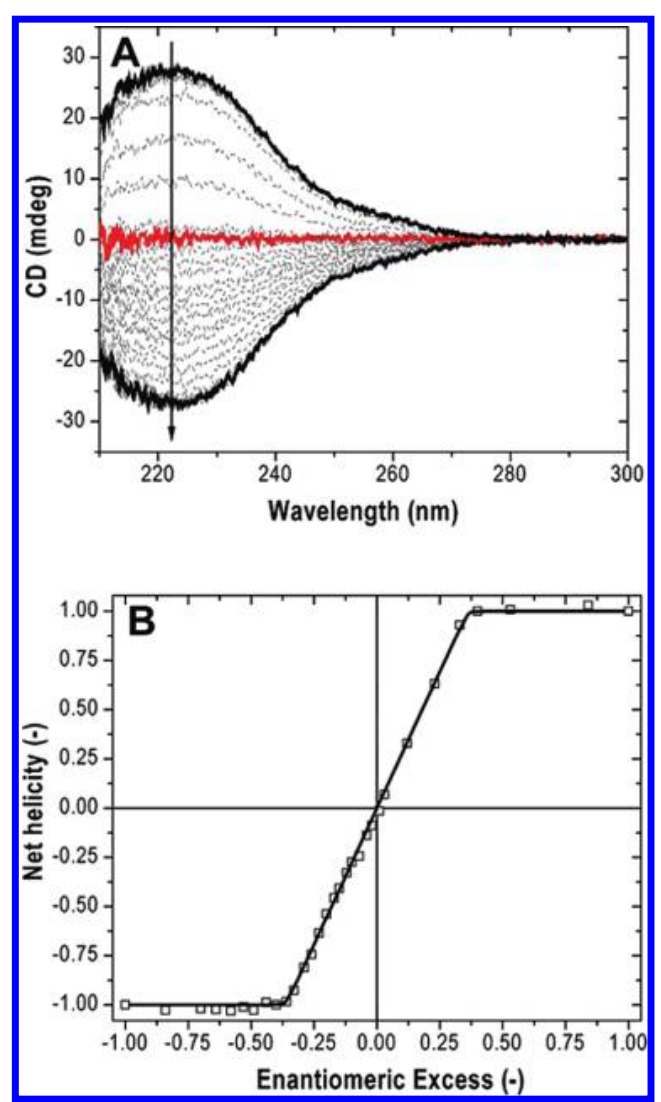

Figure 2. (A) CD spectra for mixtures of $(S)-\mathbf{1}$ and $(R)-\mathbf{1}$ recorded at $20{ }^{\circ} \mathrm{C}$. Arrow indicates the change upon going from pure $(R)-1$ to pure $(S)-\mathbf{1}$. The CD spectrum in red corresponds to the $(R)-\mathbf{1}:(S)-\mathbf{1}$ mixture with ee $=0$. (B) Net helicity as a function of the ee with the corresponding fit. For the raw CD data, see the Supporting Information. Concentration, 2.0 $\times 10^{-5} \mathrm{M}$ in $\mathrm{MCH}$.

yielded the dimensionless energy penalties, $\sigma$ and $\omega$, which are related to the HRP and the MMP via $\sigma=\exp [-2 \mathrm{HRP} / R T]$ and $\omega=\exp [-\mathrm{MMP} / R T]$, respectively. ${ }^{28}$ Modeling of the data resulted in a large HRP of $17 \mathrm{~kJ} \mathrm{~mol}^{-1}$ compared to a MMP of $1.8 \mathrm{~kJ} \mathrm{~mol}^{-1}$. Furthermore, we estimated the error in the values for $\sigma$ and $\omega$, obtained from modeling, by determining the sum of squared residuals as a function of $\sigma$ and $\omega .^{50}$ This contour plot clearly shows that we can determine the MMP, $\omega$, with good accuracy. By contrast, there is a considerable spread in the HRP, $\sigma$, whose value lies between $10^{-7}$ and $10^{-3}$, corresponding to a value of HRP between 8.4 and $20 \mathrm{~kJ} \mathrm{~mol}^{-1}$. Although there is a considerable error in the determined value for the HRP, it is still clear that it is rather high. The origin of this high value is most probably related to the high energy penalty that needs to be overcome for a helix reversal to disrupt the triple intermolecular hydrogen bonds that are present in stacks of these discotic molecules. ${ }^{30,51}$ Considering this high HRP, it is less unfavorable to "simply" incorporate chiral molecules with the "wrong" chiral side chain in a stack with a given handedness (i.e., introduce a mismatch).

This high HRP prompted us to reconsider the sergeants-andsoldiers data previously reported. ${ }^{30}$ Along with the majorityrules model, van Gestel also derived an analogous model for the sergeants-and-soldiers phenomenon, ${ }^{29}$ in which only a HRP is considered. No MMP was considered in this model, since

(51) Smulders, M. M. J.; Buffeteau, T.; Cavagnat, D.; Wolffs, M.; Schenning, A. P. H. J.; Meijer, E. W. Chirality 2008, 20, 1016-1022. the achiral "soldier" molecules do not have a preference for stacks with either handedness, while the chiral "sergeant" molecules will dictate the handedness of the stacks in which they are incorporated, meaning that they are never incorporated in a stack of their unpreferred helicity. However, majority-rules experiments showed a high HRP of more than $11 \mathrm{~kJ} \mathrm{~mol}^{-1}$ for molecules $(S)-\mathbf{1}$ and $(R)-\mathbf{1}$, which means that it is highly unfavorable to reverse the handedness of a stack. As a result of this, it could be possible for a chiral "sergeant" molecule, which is typically present in only a small fraction $(<10 \%)$, to become incorporated in a stack of achiral "soldier" molecules of its unpreferred handedness. This way, the handedness of the stack is maintained and only a relatively small MMP is paid, which is more favorable than reversing the helicity of the stack, given the high HRP.

To investigate this hypothesis, we adapted the previously reported sergeants-and-soldiers model $^{29}$ to also include a MMP ${ }^{52}$ the details of which can be found in the Supporting Information. As the statistical mechanical descriptions of the two amplification phenomena are intrinsically different, the energy penalties for each of the two phenomena are not necessarily identical. We expect, however, that the HRP must be very similar in both types of experiments, since it is related to the intermolecular hydrogen bonds. The MMP, on the other hand, has different physical meaning in the two types of experiments. For the sergeants-and-soldiers experiment a MMP arises when the chiral "sergeant" is incorporated in a stack of achiral molecules of its unpreferred helicity. In contrast, for the majority-rules experiment the MMP arises when one chiral enantiomer is incorporated in a stack formed from chiral monomers of opposite stereoconfiguration with corresponding opposite helicity.

New sergeants-and-soldiers experiments were performed with $(R)-\mathbf{1}$ and $\mathbf{2}$ in $\mathrm{MCH}$ at room temperature (Figure 3). Previously reported sergeants-and-soldiers experiments were performed in heptane, ${ }^{30,48}$ but similar results have been obtained in $\mathrm{MCH}$ (vide infra). ${ }^{53}$ Also in $\mathrm{MCH}$ we observed a strong nonlinear increase in Cotton effect upon addition of the "sergeant" solution. Already at 5\% "sergeant" the Cotton effect has saturated. Also observable in Figure $3 \mathrm{~A}$ is that the CD spectrum at low fraction of "sergeant" has a different shape, i.e., a maximum at $216 \mathrm{~nm}$, compared to the $\mathrm{CD}$ spectrum of the pure "sergeant", with a maximum at $223 \mathrm{~nm}$. This difference was attributed to a slightly different organization of the achiral molecules in stacks with a preferred handedness imposed by the "sergeant", 54 which is further discussed in the subsequent contribution (DOI 10.1021/ja9080875).

Again, we obtained a least-squares fit of the experimental sergeants-and-soldiers data shown in Figure $3 \mathrm{~B},{ }^{50}$ yielding the dimensionless energy penalties $\sigma$ and $\omega$. The energy penalties represent the same parameters as defined above for the majorityrules principle, i.e., the HRP and the MMP, respectively. We could determine the HRP with greater accuracy than previously based on the majority-rules data. The contour plot of the sum of squared residuals revealed a dimensionless HRP, $\sigma$, of about $10^{-4}$, corresponding to a helix reversal energy penalty of $11 \mathrm{~kJ}$

(52) It should be noted that we only consider a MMP for the chiral "sergeant". The MMP for the achiral "soldier" is 0, as an achiral molecule has no preference for either helicity.

(53) It was found that the solubility in $\mathrm{MCH}$ is better than in heptane, which facilitated the experiments.

(54) Stals, P. J. M.; Smulders, M. M. J.; Martín-Rapún, R.; Palmans, A. R. A.; Meijer, E. W. Chem.-Eur. J. 2009, 15, 2071-2080. 


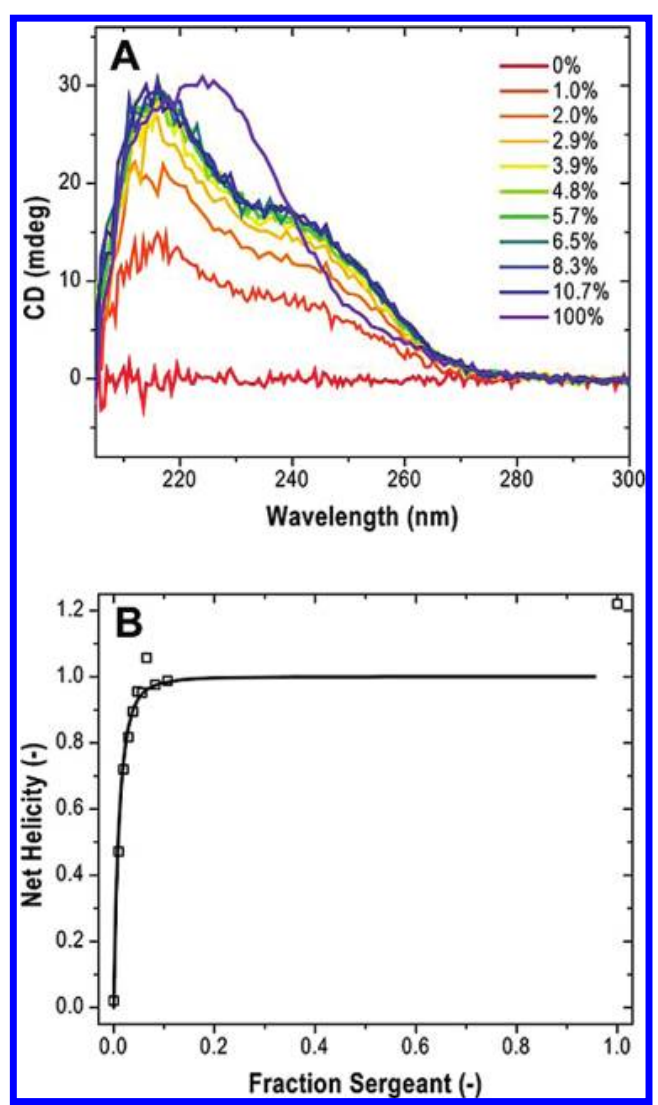

Figure 3. (A) CD spectra for mixtures of $(R)-\mathbf{1}$ and $\mathbf{2}$ recorded at $20^{\circ} \mathrm{C}$. (B) Net helicity as a function of the fraction of "sergeant" with the corresponding fit. For the raw $\mathrm{CD}$ data, see the Supporting Information. Concentration, $2.0 \times 10^{-5} \mathrm{M}$ in $\mathrm{MCH}$.

$\mathrm{mol}^{-1} .50$ The latter value is very similar to the value found for the majority-rules data, in particular when considering the uncertainty in determining the value in the latter case. This similarity is explained by the fact that for both systems equally strong intermolecular hydrogen bonds are present in the stacks. In contrast, from the sergeants-and-soldiers data, it is only possible to determine a lower limit of about $0.5 \mathrm{~kJ} \mathrm{~mol}^{-1}$ for the MMP, as can be seen in the contour plot of the sum of squared residuals. ${ }^{50}$ Considering the energy penalties and their uncertainties obtained from fitting the experimental data for both amplification phenomena, it is possible to describe both sets of data with a single value for the HRP and a single value for the MMP. That is, a HRP of about $11 \mathrm{~kJ} \mathrm{~mol}^{-1}$ and a MMP of about $1.9 \mathrm{~kJ} \mathrm{~mol}^{-1}$ result in a good fit of both data sets, as can be seen in Figures $2 \mathrm{~B}$ and $3 \mathrm{~B}$.

The above results show that there is a very strong chiral amplification behavior at room temperature for the $C_{3}$-symmetrical discotics. In this respect, these supramolecular polymers behave similarly to their covalent counterparts, like the helical polyisocyanates reported by Green and co-workers, which also display strong sergeants-and-soldiers ${ }^{9,10}$ and majority-rules effects. ${ }^{11}$ For example, analysis of the majority-rules data reported by Green ${ }^{11}$ with the van Gestel model yielded a HRP of $14.9 \mathrm{~kJ} \mathrm{~mol}^{-1}$ and a MMP of $0.23 \mathrm{~kJ} \mathrm{~mol}^{-1}$. $^{28,38}$

Effect of Temperature on Chiral Amplification. In contrast to covalent polymers, the strength of the intermolecular, noncovalent interactions present in a supramolecular polymer are strongly dependent on temperature. Therefore, we explored the effect of temperature on chiral amplification in the $C_{3^{-}}$ symmetrical discotics. Due to the noncovalent nature of the intermolecular interactions, the strength of these interactions decreases with increasing temperature, which will lead to both a decrease in the degree of aggregation and a decrease in the average stack length. However, due to the cooperative selfassembly mechanism of these BTA molecules, long stacks, exceeding 100 monomers, are predicted to be present in solution also at elevated temperatures. ${ }^{48}$

Therefore, we extended our studies to lower $\left(10{ }^{\circ} \mathrm{C}\right)$ as well as higher $\left(40\right.$ and $50{ }^{\circ} \mathrm{C}$ ) temperatures and studied both the sergeants-and-soldiers and majority-rules phenomena as a function of temperature. The results are shown in Figure 4A,B, respectively. It should be noted that with increasing temperature the maximum Cotton effect, corresponding to a fully homochiral system, decreases. This is related to the fact that fewer molecules are stacked and the average stack length decreases at higher temperature, while at the same time the fraction of monomer increases.

To compare the results obtained at different temperatures, the $\mathrm{CD}$ values were converted into the dimensionless net helicity parameter, $\eta$. This implies that we only focus on the helicity of the self-assembled molecules and we ignore the contribution of monomers. ${ }^{55}$ The net helicity versus the fraction of "sergeant" or ee determined at the different temperatures is given in Figure 4C,D. Remarkably, when comparing the two chiral amplification phenomena, we see an opposite effect of temperature on the degree of chiral amplification. For the sergeants-and-soldiers experiments, upon increasing the temperature, a higher fraction of "sergeant" is required in order to obtain a homochiral system (i.e., $\eta=1$ ). Conversely, in case of the majority-rules experiments, upon increasing the temperature, a lower ee is required in order to obtain a homochiral system. Using the same leastsquares method, we could also fit the sergeants-and-soldiers and majority-rules data and determine the helix reversal and mismatch energy penalties as a function of temperature, which are listed in Table $1 .^{50}$

When considering the results of fitting the sergeants-andsoldiers data, it can be concluded that we can determine the HRP quite accurately to a value of $10-12 \mathrm{~kJ} \mathrm{~mol}^{-1}$, which decreases only slightly with temperature. The MMP, however, can only be determined with a considerable uncertainty, and in fact it is only possible to determine a lower value for this parameter. Fortunately, the results of fitting the majority-rules data show an opposite behavior: now the MMP can be determined accurately, while the HRP shows a considerable spread. Hence, by combining the data from the two chiral amplification phenomena, it is possible to determine the energy penalties quite accurately. This shows that the HRP has a value of $10-12 \mathrm{~kJ} \mathrm{~mol}^{-1}$ and shows a small decrease with increasing temperature, whereas the MMP decreases from $2.2 \mathrm{~kJ} \mathrm{~mol}^{-1}$ (at $10{ }^{\circ} \mathrm{C}$ ) to $1.1 \mathrm{~kJ} \mathrm{~mol}^{-1}$ (at $50{ }^{\circ} \mathrm{C}$ ) when the temperature is increased. As discussed before, the MMP in the sergeants-andsoldiers experiments is not necessarily similar to the value in the majority-rules experiment. However, the decrease in strength of sergeants-and-soldiers effect upon raising the temperature, is explained by a decreasing MMP (vide infra).

These results suggest that upon raising the temperature, the intermolecular hydrogen bonds are still capable of directing the handedness of the stacks, making it energetically unfavorable for a helix reversal to occur. This is most likely also facilitated

(55) This also implies that we assume that there is no difference in distribution of the two components in the two states (aggregated and monomeric states). 


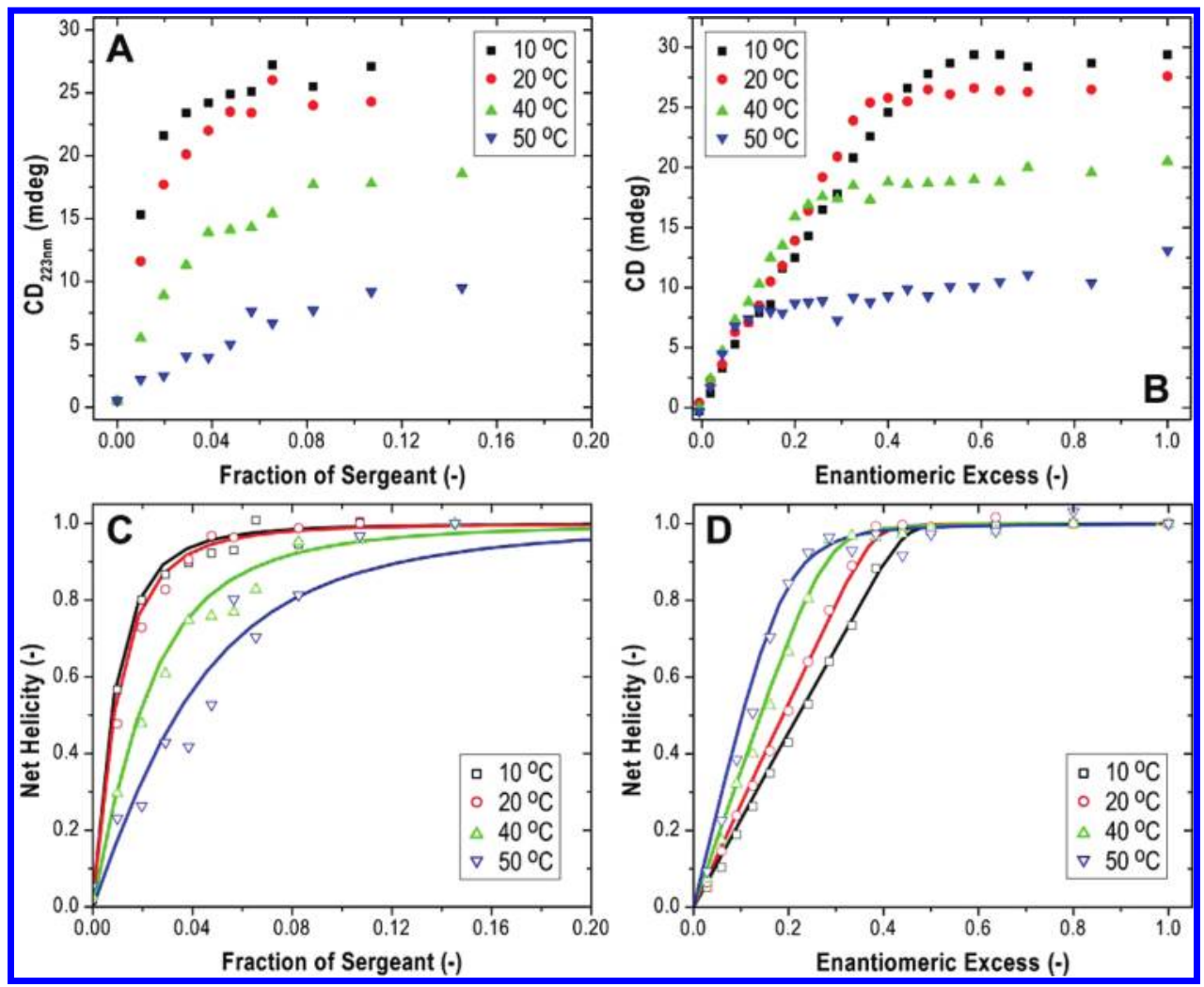

Figure 4. CD intensity at $223 \mathrm{~nm}$ versus fraction "sergeant" (A) or ee (B) and net helicity versus fraction "sergeant" (C) or ee (D) for four different temperatures: 10 (black), 20 (red), 40 (green), and $50{ }^{\circ} \mathrm{C}$ (blue). ${ }^{50} \mathrm{In}(\mathrm{C})$ and (D) the corresponding fit is also shown. Concentration, $2.0 \times 10^{-5} \mathrm{M}$ in $\mathrm{MCH}$.

Table 1. Helix Reversal Penalty and Mismatch Penalty (Both in kJ $\mathrm{mol}^{-1}$ ) at Four Temperatures, Determined from Fitting the Sergeants-and-Soldiers and Majority-Rules Data

\begin{tabular}{cccccc} 
& \multicolumn{2}{c}{ sergeants-and-soldiers } & & \multicolumn{2}{c}{ majority-rules } \\
\cline { 2 - 3 } \cline { 5 - 6 } temp $\left({ }^{\circ} \mathrm{C}\right)$ & HRP & MMP & & HRP & MMP \\
\hline 10 & $12.1 \pm 1.5$ & $>0.5^{a}$ & & $13 \pm 3$ & $2.2 \pm 0.3$ \\
20 & $12.6 \pm 2.0$ & $>0.5^{a}$ & & $15 \pm 4$ & $1.9 \pm 0.2$ \\
40 & $11.7 \pm 2.0$ & $>0.6^{a}$ & & $16 \pm 4$ & $1.5 \pm 0.2$ \\
50 & $10.2 \pm 1.9$ & $>0.6^{a}$ & & $16 \pm 3$ & $1.1 \pm 0.2$ \\
\hline
\end{tabular}

${ }^{a}$ From fitting the sergeants-and-soldiers data with the two-penalties model it could be only be concluded that $\omega$ was smaller than $\sim 0.8$. This can be seen in the contour plot sum of squared residuals as a function of $\sigma$ and $\omega .{ }^{50}$

by fact that there are three hydrogen bonds per supramolecular bond (Figure 1). Alternatively, the reducing MMP suggests that the unfavorable steric interactions of the stereogenic center present in the alkyl side chains are becoming less important at higher temperature. At higher temperature the molecules are slightly farther apart, which would reduce the unfavorable steric interactions in the side chains, and thereby lower the MMP, while the intermolecular hydrogen bonds remain intact. Furthermore, the mobility in the alkyl side chains will also be enhanced at higher temperature, increasing the possibility of attaining a more favorable conformation of the alkyl chain.

The fact that the MMP decreases with increasing temperature explains why the degree of chiral amplification is reduced in the case of the sergeants-and-soldiers principle but is enhanced in the case of the majority-rules principle. A lower MMP means that, in the former case, it becomes less unfavorable to incorporate a "sergeant" molecule in a stack of its unpreferred helicity. This means that at higher temperature a larger fraction of the "sergeant" molecules is temporarily rendered inoperative as a chiral director. Alternatively, for the majority-rules principle, a lower MMP indicates that a larger fraction of the minor enantiomer is incorporated in stacks of the major enantiomer with corresponding helicity. The main difference between the two amplification phenomena can be further explained by considering whether the major or minor component in solution dictates the helicity. Increasing the temperature will make it more favorable to incorporate the minor component, which can be either the "sergeant" or the minor enantiomer, in a stack of its unpreferred helicity. This is a disadvantageous effect in the case of the sergeants-and-soldiers principle, as the chirality is determined by the minor component in solution, i.e., the "sergeant". However, for the majority-rules principle this is advantageous, as now the major enantiomer can control a relatively larger fraction of the minor enantiomer.

These results show that there is a rather strong effect of temperature on the degree of chiral amplification and the effects of temperature on the two amplification phenomena are contrary to one another: raising the temperature decreases the sergeantsand-soldiers effect but enhances the majority-rules effect. Therefore, when quantifying chiral amplification data, it is important also to study the effects at different temperatures.

Diluted Majority-Rules Principle. The combination of the two chiral amplification phenomena, i.e., the sergeants-and-soldiers and majority-rules principles, was also investigated as function of temperature. This is of particular interest since the dependence on temperature of the two effects is opposite, i.e., upon raising the temperature, the sergeants-and-soldiers effect becomes less strong, whereas the majority-rules effect is enhanced. This combination of the two phenomena is known as the diluted majority-rules principle and has already been described for 


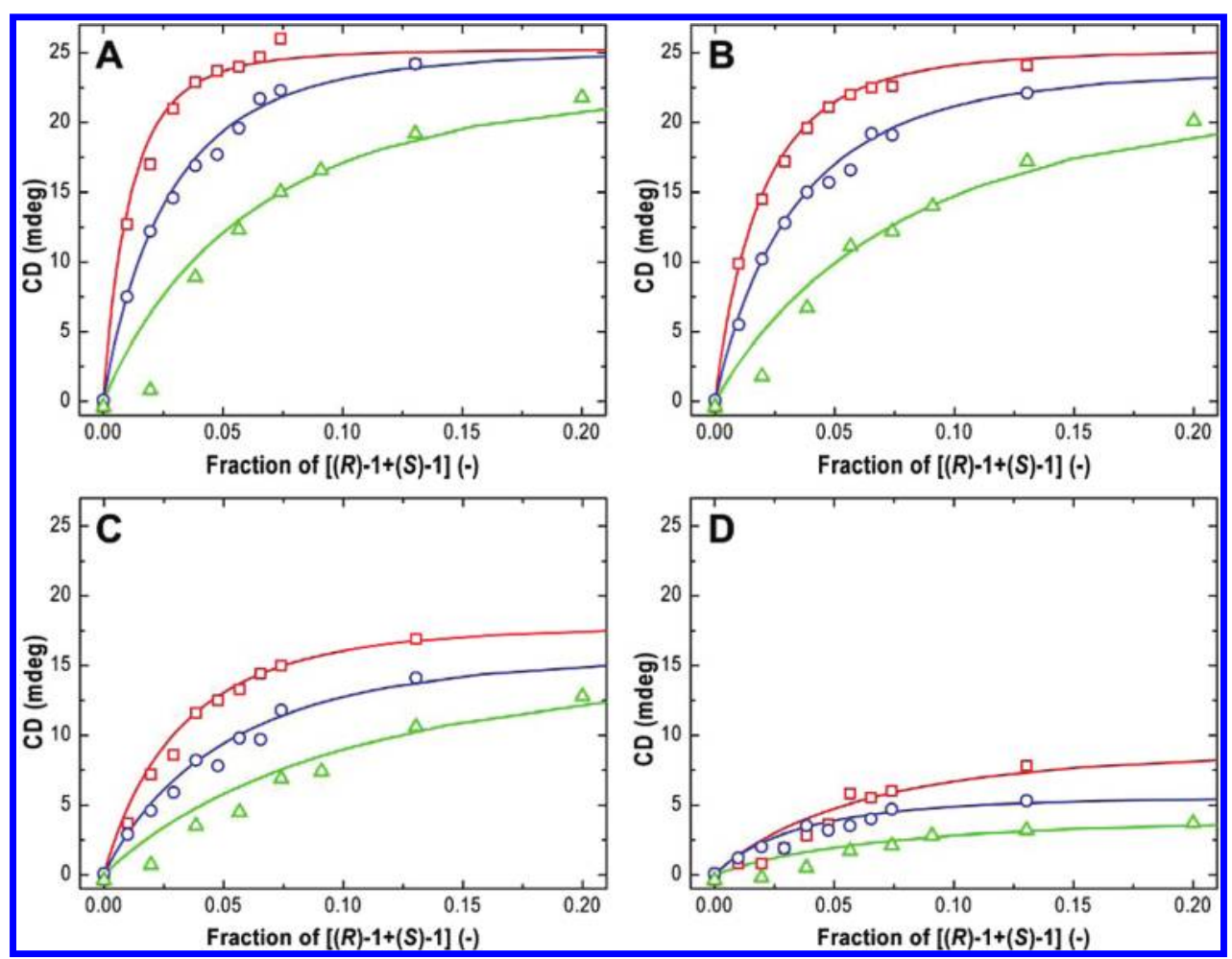

Figure 5. CD intensity at $223 \mathrm{~nm}$ versus fraction of $[(R)-\mathbf{1}+(S)-\mathbf{1}]$ for three different ee values recorded at $10(\mathrm{~A}), 20$ (B), $40(\mathrm{C})$, and $50{ }^{\circ} \mathrm{C}(\mathrm{D})$. For each temperature the ee values were 0.80 (red squares), 0.45 (blue circles), and 0.30 (green triangles). The lines are drawn to guide the eye. Concentration, 1.8 $\times 10^{-5} \mathrm{M}$ in $\mathrm{MCH}$.

covalent polymers ${ }^{23,56}$ and for supramolecular polymers, ${ }^{26,36}$ but only at one temperature.

We set out to investigate the diluted majority-rules principle as a function of temperature by adding a mixture of the two enantiomers, $(R)-\mathbf{1}$ and $(S)-\mathbf{1}$, for three different ee values, to a solution of the achiral discotic 2, performed at four different temperatures. Again we monitored the CD intensity as a function of the fraction of enantiomer mixture added (Figure 5).

For a given temperature, it is clear that lowering the ee requires that more of the enantiomer solution be added to obtain the same CD intensity, i.e., the same degree of helicity. This shows that adding the second enantiomer to a solution with an ee of $100 \%$, corresponding to a "sergeant" solution, which reduces the ee, makes the "sergeant" less efficient and hence reduces the chiral amplification behavior. Also, it can be observed that, at constant ee, raising the temperature requires that more of the enantiomer solution be added to obtain the same degree of helicity. When comparing the data at different temperatures, the fact that at higher temperature the maximum CD intensity decreases should be taken account.

For the two highest temperatures at the lowest ee, the CD intensity levels off at a lower value as compared to the higher ee values, showing that at these high temperatures an ee of only 0.30 cannot render the system completely homochiral, regardless of the amount of enantiomer solution added.

These diluted majority-rules experiments at different temperatures show that increasing the temperature results in a reduction in the degree of chiral amplification, analogous to the temperature dependence of the sergeants-and-soldiers principle. This behavior can be rationalized when considering the MMP

(56) Jha, S. K.; Cheon, K.-S.; Green, M. M.; Selinger, J. V. J. Am. Chem. Soc. 1999, 121, 1665-1673. and whether it is the major or minor component in solution that dictates the helicity. In line with the sergeants-and-soldiers principle, the chirality is determined by the minor components in solution, which are now the two enantiomers. As was discussed above, this means that, when the temperature in raised, which will lower the MMP, it becomes less unfavorable to incorporate the minor enantiomer in stacks of its unpreferred helicity. As a result, also in the case of the diluted majorityrules principle, the efficiency of chiral amplification is reduced upon increasing the temperature.

A Closer Look at the Temperature Dependence of the Majority-Rules Principle. To better understand the role of temperature in these chiral amplification studies, we studied the majority-rules phenomenon as a function of temperature in greater detail. Hence, temperature-dependent CD measurements were performed to follow the formation of self-assembled helical structures with preferred handedness. ${ }^{48}$ The CD intensity was monitored at $223 \mathrm{~nm}$ as function of temperature for different values of ee. By relating the curves for the different ee values to the curve corresponding to the pure $(R)$-enantiomer, we could determine to what extent the degree of chiral amplification, as expressed by the net helicity, $\eta$, changed with temperature. Figure 6 shows the CD intensity at $223 \mathrm{~nm}$ for different ee values as a function of temperature at a concentration of $2.5 \times$ $10^{-5} \mathrm{M}$ in $\mathrm{MCH}$. The data were obtained by heating the solution from 20 to $70{ }^{\circ} \mathrm{C}$ at a rate of $1{ }^{\circ} \mathrm{C} \mathrm{min}{ }^{-1} .57$

We observed an initial increase in $\mathrm{CD}$ intensity upon heating for the four lowest ee values (i.e., 8, 14, 21, and 33\%). With further heating the $\mathrm{CD}$ intensity then decreased again and ultimately became zero around $60{ }^{\circ} \mathrm{C}$. This is the temperature

(57) At this heating rate, no hysteresis was observed upon cooling the solution at the same rate. 


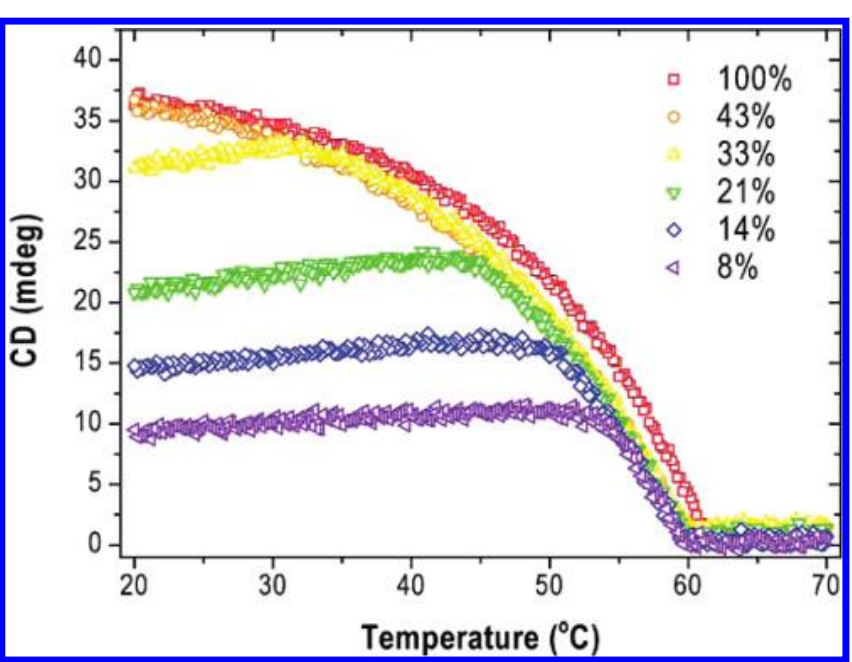

Figure 6. Temperature-dependent CD intensity at $223 \mathrm{~nm}$ for different $(S)-\mathbf{1 :}(R)-\mathbf{1}$ mixtures with different ee values. Concentration, $2.5 \times 10^{-5} \mathrm{M}$ in $\mathrm{MCH}$.

at which all self-assembled structures have disintegrated into monomers. This critical temperature, or elongation temperature, is about $2{ }^{\circ} \mathrm{C}$ lower for the mixtures of the two enantiomers than for the pure $(R)$-enantiomer $(\eta=100 \%)$, which is indicative of a slightly destabilizing effect of the minor enantiomer in the stacks of the major enantiomer. ${ }^{50}$

The increase in $\mathrm{CD}$ intensity with increasing temperature results from the reduction in MMP, which allows for a larger fraction of the minor enantiomer to be incorporated in the stacks of the major enantiomer. This will lead to a larger fraction of stacks with a preferred handedness and consequently a higher CD intensity. However, during heating part of the stacks will disassemble into monomers, which will reduce the fraction of stacks with a preferred handedness and hence also the CD intensity. At lower temperatures the first effect is dominant, leading to an increase in CD intensity, while at higher temperatures the second effect will take over and results in a rapid reduction of $\mathrm{CD}$ intensity.

Eventually, the heating curves for every ee follow the curve corresponding to the pure $(R)$-enantiomer, provided we ignore the small shift in elongation temperature (vide supra). This means that for every ee a homochiral system can be obtained by increasing the temperature. For the temperature at which this occurs, the CD effect of the mixture of enantiomers is equal to the $\mathrm{CD}$ effect of the pure $(R)$-enantiomer, meaning that all the monomers that are aggregated have become incorporated in stacks with a single helicity corresponding to the enantiomer in excess, i.e., the $(R)$-enantiomer. That is, on a supramolecular level, the aggregated fraction has reached a homochiral system as only one helicity remains. These results again show the importance of studying the temperature dependence of the chiral amplification phenomena, since for a given ee different values for the net helicity and hence also for the degree of chiral amplification can be found, depending on the temperature.

Since we are dealing with supramolecular polymers, raising the temperature will first of all affect the energy penalties, since they depend on the absolute temperature. However, the fraction of aggregated molecules decreases in favor of an increase in the monomer fraction; this is related to a temperature relative to the elongation temperature, which in turn will depend on the concentration. To determine whether only the absolute temperature influences the energy penalties, or whether the concentra- tion also plays a role, we repeated the above experiments at two different concentrations, $1.4 \times 10^{-5}$ and $3.6 \times 10^{-5} \mathrm{M}$.

For these two concentrations results similar to those depicted in Figure 6 were obtained. ${ }^{50}$ Again an initial increase in CD intensity could be observed. Also, for every ee value a temperature could be determined at which a homochiral system was obtained. The temperature at which this occurred, $T_{\text {homochiral }}$, was plotted against the corresponding ee for each concentration (Figure 7A).

The curves in Figure 7A clearly show that for a lower ee value the temperature needs to be increased further to reach homochirality, as expressed by a higher $T_{\text {homochiral, as was }}$ discussed above. Moreover, for a given ee, increasing the concentration means also that the temperature needs to be increased further to reach homochirality, which shows that also the concentration has an influence on the degree of chiral amplification. The role of concentration becomes even clearer when we plot the relative temperature, defined as the difference between $T_{\text {homochiral }}$ and $T_{\mathrm{e}}$, versus the ee for the three concentrations (Figure 7B). Now, the data for the three concentrations nicely line up and there is an almost linear relationship between the relative temperature, $T_{\mathrm{e}}-T_{\text {homochiral }}$, and the ee. The fact that the relative temperature is controlling the degree of chiral amplification was further confirmed by the construction of a "master curve", in which the normalized CD intensity, $\phi,{ }^{50}$ is plotted versus the dimensionless, relative temperature, $T / T_{\mathrm{e}}$ (Figure 8). The curve in Figure 8 is independent of the absolute temperature or concentration. It is only when we select a certain concentration, which will have a certain corresponding elongation temperature, $T_{\mathrm{e}}$, that we can construct an $x$-axis with absolute temperature. Independent of the concentration, a decreasing ee leads to a higher $T_{\text {homochiral }}$ and a broader temperature range over which the normalized $\mathrm{CD}$ intensity increases.

The above results strongly suggest that the mechanism that controls the degree of chiral amplification in the case of the majority-rules principle is mostly dependent on the relative temperature, that is, the difference between the actual temperature and the elongation temperature. Two parameters that also depend on this relative temperature are the free monomer concentration and the average stack length. The former decreases below the elongation temperature, whereas the latter increases below the elongation temperature. In fact, since these two parameters are related, both parameters are most likely involved in the mechanism that controls the degree of chiral amplification.

\section{Conclusion}

It was found that strong amplification of chirality is observed in supramolecular polymers consisting of benzene-1,3,5-tricarboxamide monomers. The equilibrium between monomers and helical stacks (supramolecular polymers) is under thermodynamic control with a strong helical architecture based on triple hydrogen bonding, which makes the system ideally suited for detailed stereochemical studies. To quantify the chiral amplification phenomena, we adapted the previously reported sergeantsand-soldiers model. This allowed us to describe both phenomena in terms of two energy penalties: the helix reversal penalty and the mismatch penalty. The former was ascribed to the formation of intermolecular hydrogen bonds and was found to be rather high. The latter energy penalty was related to steric interactions in the alkyl side chains due to the stereogenic center present. Upon raising the temperature, the helix reversal penalty remained rather constant, showing that the intermolecular 


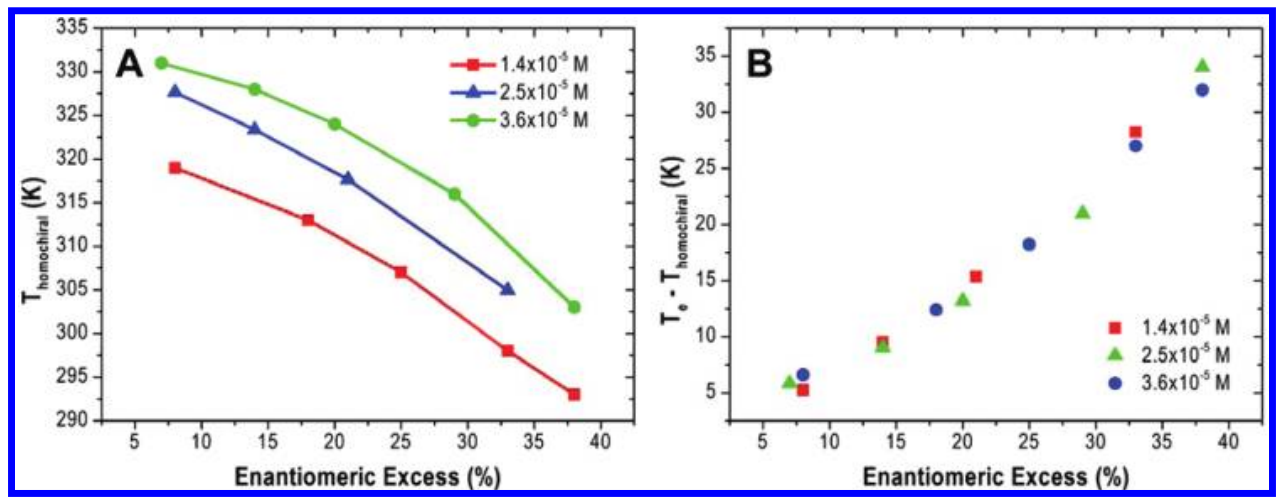

Figure 7. (A) Temperature at which homochirality is reached, $T_{\text {homochiral }}$. (B) Relative temperature with respect to the elongation temperature, $T_{\mathrm{e}}-T_{\text {homochiral }}$, versus ee for three concentrations.

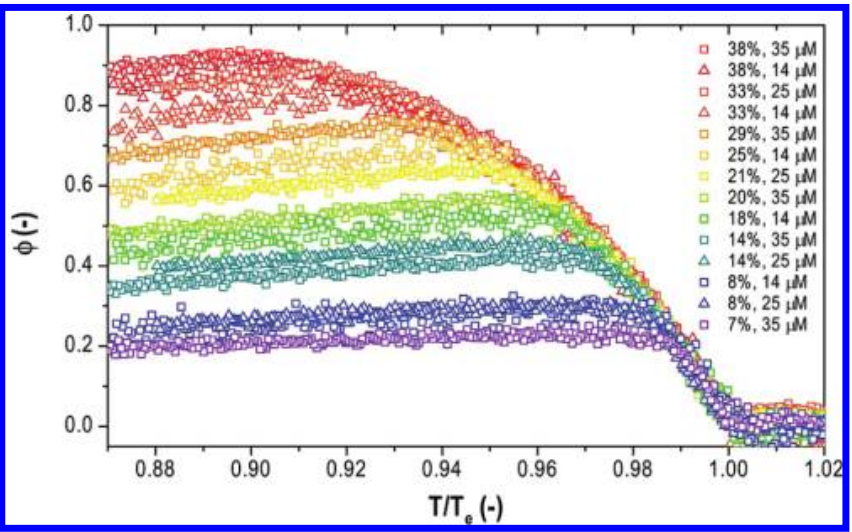

Figure 8. Master curve obtained from plotting the normalized CD effect versus the dimensionless temperature, $T / T_{\mathrm{e}}$.

hydrogen bonds remain intact and are directing the helicity in the stack. The mismatch penalty does, however, decrease when the temperature is increased, which resulted in opposite effects on the degree of chiral amplification when comparing the sergeants-and-soldiers and the majority-rules experiments. While for the former a reduction in mismatch penalty resulted in a decrease in degree of chiral amplification, for the latter it resulted in a stronger chiral amplification effect. The opposing behavior could be rationalized by considering whether the major or minor component in solution dictates the helicity of the stack. By combining the sergeants-and-soldiers and majority-rules phenomena in a diluted majority-rules experiment, we could further determine the effect of temperature on the degree of chiral amplification. Extending the experiments to different concentrations revealed that the relative temperature, i.e., the temperature relative to the critical temperature of elongation, controls the degree of chiral amplification. As a result, it was possible to generate a general "master curve" independent of concentration to describe the temperature-dependent majority-rules principle. For further understanding of the amplification phenomena, a kinetic simulation, making use of the Gillespie methodology, is currently being developed in our laboratories. This simulation allows for the determination of the distribution of the two enantiomers over the aggregated and the molecular dissolved states. Full analysis of this method to kinetically describe the amplification phenomena is in progress and will be published soon.

In conclusion, these findings show that the extended chiral amplification model allows for a detailed study of the temperature dependence of chiral amplification phenomena and can yield insights into how to exert control over the degree of chiral amplification. In addition, because the degree of chiral amplification depends strongly on temperature, and to a lesser extent on concentration, the effect of these parameters on the chiral amplification phenomena should be considered for a proper characterization of the chiral amplification behavior.

Acknowledgment. We thank Prof. Jim Feast for scientific discussions. The authors thank the National Research School Combination Catalysis (NRSC-Catalysis) for funding.

Supporting Information Available: Experimental conditions, details about the amplification model, modeling procedures, and supporting figures and tables. This material is available free of charge via the Internet at http://pubs.acs.org.

JA908053D 